\title{
On the controllability of a free-boundary problem for the 1D heat equation
}

\author{
E. Fernández-Cara* ${ }^{*}$ J. Limaco ${ }^{\dagger}$, S. B. de Menezes ${ }^{\ddagger}$
}

\begin{abstract}
This paper deals with the local null control of a free-boundary problem for the classical $1 \mathrm{D}$ heat equation with distributed controls, locally supported in space. In the main result we prove that, if the final time $T$ is fixed and the initial state is sufficiently small, there exist controls that drive the state exactly to rest at time $t=T$.
\end{abstract}

Keywords: null controllability, free-boundary problems, 1D heat equation, Carleman estimates. Mathematics Subject Classification: 93B05, 93C20, 35K20, 35R35.

\section{Introduction. The main result}

Assume that $L_{0}>0, T>0,0<a<b<L_{*}<L_{0}$ and $y_{0} \in L^{\infty}\left(0, L_{0}\right)$ are given. In this paper, we consider free-boundary problems of the following kind:

Find $L \in C^{1}([0, T])$ with $L(t)>0$ for all $t$ and a function $y=y(x, t)$ such that

$$
\begin{gathered}
L(0)=L_{0}, \\
\left\{\begin{array}{l}
y_{t}-y_{x x}=v 1_{\omega}, \quad(x, t) \in Q_{L}, \\
y(0, t)=0, \quad y(L(t), t)=0, \quad t \in(0, T), \\
y(x, 0)=y_{0}(x), \quad x \in\left(0, L_{0}\right)
\end{array}\right.
\end{gathered}
$$

and, moreover,

$$
y_{x}(L(t), t)=-L^{\prime}(t), \quad t \in(0, T) .
$$

Here, we have used the notation

$$
Q_{L}=\{(x, t): x \in(0, L(t)), \quad t \in(0, T)\}, \quad \omega=(a, b) ;
$$

*Dpto. E.D.A.N., Universidad de Sevilla, Aptdo. 1160, 41080 Sevilla, Spain, cara@us.es. Partially supported by grant MTM2013-41286-P (DGI-MINECO, Spain) and CAPES (Brazil)

${ }^{\dagger}$ Inst. Matemática - Universidade Federal Fluminense, RJ, Brasil, jlimaco@vm.uff.br.

${ }^{\ddagger}$ Dpto. Matemática - Universidade Federal do Ceará, CE, Brasil, silvano@mat.ufc.br. 
as usual, $1_{\omega}$ denotes the characteristic function of $\omega$.

In (1)-(3), $v$ is a control and $(L, y)$ is an associated state. Recall that, for any $y_{0} \in H_{0}^{1}\left(0, L_{0}\right)$ and $v \in L^{2}(\omega \times(0, T))$, there exists at least one local in time solution to (2)-(3). In other words, there exist $T_{*} \in(0, T]$ and a couple $(L, y)$ with

$$
\left\{\begin{array}{l}
L \in C^{1}\left(\left[0, T_{*}\right)\right), \quad L(t) \geq L_{*} \text { in }\left[0, T_{*}\right), \\
y, y_{x}, y_{x x}, y_{t} \in L^{2}\left(Q_{L}^{*}\right), \text { with } Q_{L}^{*}:=\left\{(x, t): x \in(0, L(t)), \quad t \in\left(0, T_{*}\right)\right\} \\
L(0)=L_{0}, \quad y(x, 0)=y_{0}(x) \text { in }\left(0, L_{0}\right)
\end{array}\right.
$$

such that the PDE in (2) is satisfied in $Q_{L}^{*}$ in the distributional sense and the boundary conditions and (3) are fulfilled in $\left(0, T_{*}\right)$. Furthermore, either $T_{*}=T$, or at least one of the following identities hold:

$$
\lim _{t \rightarrow T_{*}^{-}} L(t)=b \quad \text { or } \quad \lim _{t \rightarrow T_{*}^{-}}\left|L^{\prime}(t)\right|=+\infty .
$$

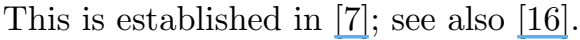

Free-boundary problems similar to (1)-(3) are motivated by many different applications:

- Solidification processes and, in particular, the so called Stefan problem, see 11, 12.

- The analysis and computation of free surface flows, see [18, 22, 25.

- Fluid-solid interaction, see 3, 21, 24.

- Gas flow through porous media, see [1, 6, 23.

- Tumor growth and other problems from mathematical biology, see [13, 14, etc.

Note that, for a fixed control $v,(1)$ and $(2)$ are not enough to identify the state $(L, y)$. We need an additional information and this is furnished by (3). In many of these areas, this condition is completely natural. For instance, in tumor growth modelling, $y$ can be viewed as a pressure (cells are pushed towards the low density regions) and (3) says that, on the moving tumor boundary, the growth speed is proportional to the pressure gradient. Of course, this is a version of the well known Darcy's law for porous media.

The main goal in this paper is to analyze the null controllability of (1)-(3). By definition, it will be said that (1)-(3) is null-controllable if, for any $y_{0} \in H_{0}^{1}\left(0, L_{0}\right)$, there exist a control $v \in L^{2}(\omega \times(0, T))$, a function $L \in C^{1}([0, T])$ and an associated solution $y=y(x, t)$ satisfying (1)(3) and

$$
y(x, T)=0, \quad x \in(0, L(T)) .
$$

Notice that, if $L$ and $(v, y)$ solve (1)-(4) and (for instance) $y(\cdot, T) \in H^{2}(0, L(T))$, then $L^{\prime}(T)=$ 0 . Consequently, the null controllability of (1)-(3) is a useful property from the viewpoint of control theory: roughly speaking, if it is fulfilled, we only have to "work" during a finite time interval in order to get the desired behavior of the system.

The controllability of linear and nonlinear PDEs has been the objective of a lot of papers the last years. In the context of the linear and semilinear heat equation, the main contributions have been 
obtained in $\underline{8,20,5,15,10,4}$. On the other hand, to our knowledge, for parabolic free-boundary problems, controllability questions have not been considered in depth; see however $\underline{2}$, 17 .

In the sequel, $C$ denotes a generic positive constant; $C_{0}, C_{1}$, etc. are other positive (specific) constants; when it makes sense, the extension by zero in space of any function $f$ is denoted by $f^{*}$.

The main result in this paper is the following:

Theorem 1.1 Let us assume that $L_{0}>0, T>0$ and $0<a<b<L_{*}<L_{0}<B$ are given. Then (1)-(3) is locally null-controllable. More precisely, there exists $\varepsilon>0$ such that, if $y_{0} \in H_{0}^{1}\left(0, L_{0}\right)$ and $\left\|y_{0}\right\|_{H_{0}^{1}\left(0, L_{0}\right)} \leq \varepsilon$, there exist $L$ and $(v, y)$, with

$$
L \in C^{1}([0, T]), \quad L_{*} \leq L(t) \leq B, \quad v \in L^{2}(\omega \times(0, T)), \quad y^{*} \in C^{0}\left([0, T] ; H_{0}^{1}(0, B)\right),
$$

that satisfy (1)-(3) and (4).

The plan of the proof is the following:

- First, we prove that there exists $\varepsilon>0$ such that, whenever $\left\|y_{0}\right\|_{H_{0}^{1}\left(0, L_{0}\right)} \leq \varepsilon$, for each $\beta>0$ there exist uniformly bounded $L_{\beta}$ and $\left(v_{\beta}, y_{\beta}\right)$ satisfying (1)-(3) and

$$
\left\|y_{\beta}(\cdot, T)\right\|_{L^{2}(0, L(T))} \leq \beta
$$

This is achieved by solving an appropriate fixed point equation in a closed convex set $\mathcal{M} \subset$ $C^{1}([0, T])$ :

$$
L=\Lambda_{\beta}(L), \quad L \in \mathcal{M}
$$

- Then, we take limits as $\beta \rightarrow 0$. Thus, from the estimates deduced for $L_{\beta}$ and $\left(v_{\beta}, y_{\beta}\right)$, we see that, at least for a subsequence, we have

$$
\begin{aligned}
& L_{\beta} \rightarrow L \text { strongly in } C^{1}([0, T]), \\
& v_{\beta} \rightarrow v \text { weakly in } L^{2}(\omega \times(0, T)), \\
& y_{\beta}^{*} \rightarrow y^{*} \text { strongly in } C^{0}\left([0, T] ; H_{0}^{1}(0, B)\right),
\end{aligned}
$$

where $(v, y)$ is a control-state pair satisfying (1)-(3) and (4).

The rest of this paper is organized as follows.

In Section 2 , we prove that, for any $L \in C^{1}([0, T])$ satisfying

$$
L_{*} \leq L \leq B, \quad L(0)=L_{0},
$$

the linear system (2) is approximately and null-controllable. We also prove that the solutions to (2) satisfy an additional regularity property. Section 3 is devoted to prove Theorem 1.1. In Section 4 we present some additional comments and we give some indications on future work. Finally, Section 5 is an Appendix where we sketch the proof of a Carleman estimate. 


\section{Some controllability results for the classical heat equation in a non-cylindrical domain}

In this section, we assume that $L_{0}>0, T>0$ and $0<a<b<L_{*}<L_{0}<B$ are given.

We fix $y_{0} \in H_{0}^{1}\left(0, L_{0}\right)$ and we assume that $L \in C^{1}([0, T])$ is a prescribed function satisfying (6); in particular, note that $L(t)>b$ for all $t \in[0, T]$.

Throughout this paper, we will use the notation

$$
N_{L}:=\left\|L^{\prime}\right\|_{\infty}, \quad N_{0}:=\left\|y_{0}\right\|_{H_{0}^{1}\left(0, L_{0}\right)} .
$$

\subsection{The problems and the results}

Let us consider the linear system

$$
\left\{\begin{array}{l}
y_{t}-y_{x x}=v 1_{\omega}, \quad(x, t) \in Q_{L}, \\
y(0, t)=0, \quad y(L(t), t)=0, \quad t \in(0, T), \\
y(x, 0)=y_{0}(x), \quad x \in\left(0, L_{0}\right) .
\end{array}\right.
$$

For every $v \in L^{2}(\omega \times(0, T))$ and every $y_{0} \in H_{0}^{1}\left(0, L_{0}\right)$, there exists exactly one solution to (8), with

$$
y, y_{x}, y_{x x}, y_{t} \in L^{2}\left(Q_{L}\right)
$$

and, consequently,

$$
y^{*} \in C^{0}\left([0, T] ; H_{0}^{1}(0, B)\right) .
$$

Indeed, an appropriate change of variable allows to rewrite (8) as a similar problem for a parabolic PDE of the form

$$
z_{\tau}-z_{\xi \xi}+h(\xi, \tau) z_{\xi}=w
$$

in a cylindrical domain, with a bounded coefficient $h$ and a square-integrable right hand side $w$; see the precise definitions of $\xi$ and $\tau$ below, after 21.

The following controllability result is satisfied by 8 :

Theorem 2.1 For any $y_{0} \in H_{0}^{1}\left(0, L_{0}\right)$ and any $\beta>0$ there exist pairs $\left(v_{\beta}, y_{\beta}\right)$, with

$$
v_{\beta} \in L^{2}(\omega \times(0, T)), \quad y_{\beta}^{*} \in C^{0}\left([0, T] ; H_{0}^{1}(0, B)\right),
$$

satisfying (8) and

$$
\left\|y_{\beta}(\cdot, T)\right\|_{L^{2}(0, L(T))} \leq \beta .
$$

Furthermore, $v_{\beta}$ can be found such that

$$
\left\|v_{\beta}\right\|_{L^{2}(\omega \times(0, T))} \leq C_{1}\left\|y_{0}\right\|_{H_{0}^{1}\left(0, L_{0}\right)},
$$

where $C_{1}$ depends on $N_{L}, L_{*}, B, \omega$ and $T$, but is independent of $\beta$.

An immediate consequence is the null controllability of (8): 
Corollary 2.1 For any $y_{0} \in H_{0}^{1}\left(0, L_{0}\right)$, there exist pairs $(v, y)$, with

$$
v \in L^{2}(\omega \times(0, T)), \quad y^{*} \in C^{0}\left([0, T] ; H_{0}^{1}(0, B)\right),
$$

satisfying (8) and (4). Furthermore, $v$ can be found such that

$$
\|v\|_{L^{2}(\omega \times(0, T))} \leq C_{1}\left\|y_{0}\right\|_{H_{0}^{1}\left(0, L_{0}\right)},
$$

where $C_{1}$ only depends on $N_{L}, L_{*}, B, \omega$ and $T$.

The proof of Theorem 2.1 follows rather standard arguments. The main tool is a global Carleman estimate for the solution to the adjoint system of (8), that is given by

$$
\left\{\begin{array}{l}
-\varphi_{t}-\varphi_{x x}=g(x, t) \quad(x, t) \in Q_{L} \\
\varphi(0, t)=0, \quad \varphi(L(t), t)=0, \quad t \in(0, T) \\
\varphi(x, T)=\varphi^{T}(x), \quad x \in(0, L(T))
\end{array}\right.
$$

where $g \in L^{2}\left(Q_{L}\right)$ and $\varphi^{T} \in L^{2}(0, L(T))$.

This will be established in the next section.

\section{$2.2 \quad$ A Carleman estimate}

In the sequel, we will denote by $\Sigma_{L}$ the lateral boundary of $Q_{L}$ :

$$
\Sigma_{L}=\{(x, t): x=0 \text { or } x=L(t), 0<t<T\} .
$$

We begin with a simple result:

Lemma 2.1 Let $\omega_{0}$ be a non-empty open set with $\overline{\omega_{0}} \subset \omega$. There exists a function $\alpha_{0} \in C^{1}\left(\overline{Q_{L}}\right)$ with $\alpha_{0, x x} \in C^{0}\left(\overline{Q_{L}}\right)$ such that

$$
\left\{\begin{array}{l}
\alpha_{0}(x, t)=0 \quad \forall(x, t) \in \Sigma_{L} \\
\left|\alpha_{0, x}\right|>0 \text { in } \overline{Q_{L}} \backslash\left(\omega_{0} \times(0, T)\right) \text { and } \\
\alpha_{0}(x, t)=1-\frac{x-b}{L(t)-b} \forall x \in(b, L(t)), \quad \forall t \in[0, T]
\end{array}\right.
$$

For the proof, it suffices to take (for instance)

$$
\alpha_{0}(x, t):= \begin{cases}\frac{x}{a}, & \text { if } 0 \leq x<a, \\ 1+p\left(\frac{2(x-a)}{b-a}, \frac{b-a}{2 a}\right), & \text { if } a \leq x<\frac{a+b}{2}, \\ 1+p\left(\frac{2(b-x)}{b-a}, \frac{b-a}{2(L(t)-b)}\right), & \text { if } \frac{a+b}{2} \leq x<b, \\ \frac{L(t)-x}{L(t)-b}, & \text { if } b \leq x \leq L(t),\end{cases}
$$

where

$$
p(w, z):=z w+(10-6 z) w^{3}+(8 z-15) w^{4}+(6-3 z) w^{5} .
$$


In particular, notice that $\left|\alpha_{0, x}\right| \geq \eta$ in $\overline{Q_{L}} \backslash\left(\omega_{0} \times(0, T)\right)$, where

$$
\frac{1}{\eta}=\max \left\{a, \max _{0 \leq t \leq T}(L(t)-b)\right\} .
$$

Let us introduce the weights $\xi$ and $\alpha$ and the functions $\alpha_{1}$ and $\gamma$, with

$$
\xi(x, t):=\frac{e^{\lambda \alpha_{1}(x, t)}}{\gamma(t)}, \alpha(x, t):=\frac{e^{2 \lambda\left\|\alpha_{1}\right\|_{\infty}}-e^{\lambda \alpha_{1}(x, t)}}{\gamma(t)}, \alpha_{1}(x, t):=\alpha_{0}(x, t)+1, \gamma(t):=t^{k}(T-t)^{k},
$$

where $\lambda>0$ and $k \geq 2$ are real numbers.

We will present now a Carleman estimate for the solution $\varphi$ of the adjoint system (12). It is inspired by the ideas in Fursikov-Imanovilov $\underline{15}$ and is contained in the following theorem:

Theorem 2.2 Let $\alpha_{0}, \alpha, \gamma$ and $\xi$ be the functions defined above. There exist positive constants $\lambda_{0}, s_{0}$ and $C_{0}$, only depending on $N_{L}, L_{*}, B, \omega$ and $T$, such that, for any $s \geq s_{0}$, any $\lambda \geq \lambda_{0}$, any $g \in L^{2}\left(Q_{L}\right)$ and any $\varphi^{T} \in L^{2}(0, L(T))$, one has

$$
\begin{gathered}
\iint_{Q_{L}} e^{-2 s \alpha}\left[(s \xi)^{-1}\left(\left|\varphi_{t}\right|^{2}+\left|\varphi_{x x}\right|^{2}\right)+\lambda^{2}(s \xi)\left|\varphi_{x}\right|^{2}+\lambda^{4}(s \xi)^{3}|\varphi|^{2}\right] d x d t \\
+\int_{0}^{T} e^{-2 s \alpha(L(t), t)} \lambda s \xi(L(t), t)\left|\varphi_{x}(L(t), t)\right|^{2} d t+\int_{0}^{T} e^{-2 s \alpha(0, t)} \lambda s \xi(0, t)\left|\varphi_{x}(0, t)\right|^{2} d t \\
\quad \leq C_{0}\left(\iint_{Q_{L}} e^{-2 s \alpha}|g|^{2} d x d t+\iint_{\omega \times(0, T)} e^{-2 s \alpha} \lambda^{4}(s \xi)^{3}|\varphi|^{2} d x d t\right),
\end{gathered}
$$

where $\varphi$ is the corresponding solution to 12 .

The proof is given in the Appendix (see Section 5).

\subsection{An observability inequality and the controllability properties of (8)}

The first consequence of Theorem 2.2 is an observability inequality. We will consider the homogeneous adjoint system

$$
\left\{\begin{array}{l}
-\varphi_{t}-\varphi_{x x}=0, \quad(x, t) \in Q_{L} \\
\varphi(0, t)=0, \quad \varphi(L(t), t)=0, \quad t \in(0, T) \\
\varphi(x, T)=\varphi^{T}(x), \quad x \in(0, L(T))
\end{array}\right.
$$

where $\varphi^{T} \in L^{2}(0, L(T))$.

Proposition 2.1 There exists $C_{2}>0$, only depending on $N_{L}, L_{*}, B, \omega$ and $T$, such that, for any $\varphi^{T} \in L^{2}(0, L(T))$, the associated solution to 14 satisfies

$$
\int_{0}^{L_{0}}|\varphi(x, 0)|^{2} d x \leq C_{2} \iint_{\omega \times(0, T)}|\varphi|^{2} d x d t .
$$

Proof: Let us take $\lambda=\lambda_{0}$ and $s=s_{0}$ in (13). Then

$$
\iint_{Q_{L}} e^{-2 s_{0} \alpha} \xi^{3}|\varphi|^{2} d x d t \leq C \iint_{\omega \times(0, T)} e^{-2 s_{0} \alpha} \xi^{3}|\varphi|^{2} d x d t
$$


and, consequently,

$$
\int_{T / 4}^{3 T / 4} \int_{0}^{L(t)}|\varphi|^{2} d x d t \leq C \int_{T / 4}^{3 T / 4} \int_{0}^{L(t)} e^{-2 s_{0} \alpha} \xi^{3}|\varphi|^{2} d x d t \leq C \iint_{\omega \times(0, T)}|\varphi|^{2} d x d t .
$$

On the other hand, by multiplying the PDE in (14) by $\varphi$ and integrating in $(0, L(t))$, we get the identities

$$
-\frac{1}{2} \frac{d}{d t}\left(\int_{0}^{L(t)}|\varphi|^{2} d x\right)+\frac{1}{2} L^{\prime}(t)|\varphi(L(t), t)|^{2}+\int_{0}^{L(t)}\left|\varphi_{x}\right|^{2} d x=0 \quad \forall t \in(0, T) .
$$

Since $\varphi(L(t), t) \equiv 0$, we deduce that

$$
\frac{d}{d t}\left(\int_{0}^{L(t)}|\varphi(x, t)|^{2} d x\right) \geq 0
$$

and, integrating in time, we deduce that

$$
\int_{0}^{L(0)}|\varphi(x, 0)|^{2} d x \leq \int_{0}^{L(t)}|\varphi(x, t)|^{2} d x \quad \forall t \in(0, T)
$$

and

$$
\frac{T}{2} \int_{0}^{L(0)}|\varphi(x, 0)|^{2} d x \leq \int_{T / 4}^{3 T / 4} \int_{0}^{L(t)}|\varphi(x, t)|^{2} d x d t .
$$

From (16) and (17), we find $(15)$ and the proof is done.

By duality, using classical arguments, it is not difficult to deduce from Proposition 2.1 the controllability result in Theorem 2.1. see $\underline{5}$. Thus, let $y_{0} \in H_{0}^{1}\left(0, L_{0}\right)$ and $\beta>0$ be given and let us introduce the function $J_{L, \beta}$, with

$$
\left\{\begin{array}{l}
J_{L, \beta}\left(\varphi^{T}\right):=\frac{1}{2} \iint_{\omega \times(0, T)}|\varphi|^{2} d x d t+\beta\left\|\varphi^{T}\right\|_{L^{2}(0, L(T))}+\left(\varphi(\cdot, 0), y_{0}\right)_{L^{2}\left(0, L_{0}\right)} \\
\forall \varphi^{T} \in L^{2}(0, L(T))
\end{array}\right.
$$

where, for each $\varphi^{T} \in L^{2}(0, L(T))$, we have denoted by $\varphi$ the unique solution to (14). Then, $J_{L, \beta}$ possesses a unique minimizer $\hat{\varphi}_{\beta}^{T}$ and the "best" control is given by

$$
v_{\beta}=\left.\hat{\varphi}_{\beta}\right|_{\omega \times(0, T)},
$$

where $\hat{\varphi}_{\beta}$ is the solution to 14 associated to $\hat{\varphi}_{\beta}^{T}$.

Furthermore, from the inequality $J_{L, \beta}\left(\hat{\varphi}_{\beta}^{T}\right) \leq J_{L, \beta}(0)=0$ and (15), we deduce that

$$
\begin{array}{rl}
\frac{1}{2} \iint_{\omega \times(0, T)}\left|\hat{\varphi}_{\beta}\right|^{2} & d x d t+\beta\left\|\hat{\varphi}_{\beta}^{T}\right\|_{L^{2}(0, L(T))} \\
\leq & -\left(\hat{\varphi}_{\beta}(\cdot, 0), y_{0}\right)_{L^{2}\left(0, L_{0}\right)} \\
& \leq \frac{1}{4 C_{2}}\left\|\hat{\varphi}_{\beta}(\cdot, 0)\right\|_{L^{2}\left(0, L_{0}\right)}^{2}+C_{2}\left\|y_{0}\right\|_{L^{2}\left(0, L_{0}\right)}^{2} \\
& \leq \frac{1}{4} \iint_{\omega \times(0, T)}\left|\hat{\varphi}_{\beta}\right|^{2} d x d t+C_{2}\left\|y_{0}\right\|_{L^{2}\left(0, L_{0}\right)}^{2}
\end{array}
$$


and, consequently,

$$
\left\|v_{\beta}\right\|_{L^{2}(\omega \times(0, T))}^{2} \leq \iint_{\omega \times(0, T)}\left|\hat{\varphi}_{\beta}\right|^{2} d x d t+2 \beta\left\|\hat{\varphi}_{\beta}^{T}\right\|_{L^{2}(0, L(T))} \leq 4 C_{2}\left\|y_{0}\right\|_{L^{2}\left(0, L_{0}\right)}^{2} .
$$

This way, we find the estimates (10) and (11), where the constant $C_{1}$ only depends on $N_{L}, L_{*}$, $B, \omega$ and $T$; see for instance $\underline{15,9]}$ for more details.

\subsection{A regularity property}

Let $(v, y)$ be a control-state pair furnished by Theorem 2.1 or Corollary 2.1 and let us introduce the set

$$
R_{L}:=Q_{L} \cap\left\{(x, t): x>b^{\prime}\right\},
$$

where $b<b^{\prime}<L_{*}$. We will see in this section that, for some $\kappa \in(0,1 / 2]$ only depending on $N_{L}$, $L_{*}, B, \omega, T$ and $N_{0}$, one has

$$
y \in C_{x, t}^{1+\kappa, \kappa / 2}\left(\overline{R_{L}}\right)
$$

where $C_{x, t}^{1+\kappa, \kappa / 2}\left(\overline{R_{L}}\right)$ is the space of functions $z \in C^{0}\left(\overline{R_{L}}\right)$ that possess continuous partial derivatives with respect to $x$ in $\overline{R_{L}}$ and satisfy

$$
\sup _{(x, t),\left(x^{\prime}, t^{\prime}\right) \in \overline{R_{L}}}\left(\frac{\left|z(x, t)-z\left(x^{\prime}, t^{\prime}\right)\right|}{\left|x-x^{\prime}\right|+\left|t-t^{\prime}\right|^{\kappa / 2}}+\frac{\left|z_{x}(x, t)-z_{x}\left(x^{\prime}, t^{\prime}\right)\right|}{\left|x-x^{\prime}\right|^{\kappa}+\left|t-t^{\prime}\right|^{\kappa / 2}}\right)<+\infty .
$$

Indeed, let $\delta>0$ be sufficiently small to have $b<b^{\prime}-\delta<b^{\prime}+\delta<L_{*}$. In view of Theorems 10.1 and 11.1 in $\underline{19}$, pp. 204-211, there exists $\kappa \in(0,1 / 2]$ depending on $N_{L}, L_{*}, B, \omega, T$ and $N_{0}$ such that

$$
y \in C_{x, t}^{1+\kappa, \kappa / 2}\left(\left[b^{\prime}-\delta, b^{\prime}+\delta\right] \times[0, T]\right) .
$$

Let us now introduce the following change of variables in $R_{L}$ :

$$
\xi=\frac{1}{L(t)-b^{\prime}}\left(b^{\prime}(L(t)-x)+L_{0}\left(x-b^{\prime}\right)\right), \quad \tau=\left(L_{0}-b^{\prime}\right)^{2} \int_{0}^{t} \frac{1}{\left(L(t)-b^{\prime}\right)^{2}} d s
$$

and let us set $z(\xi, \tau):=y(x, t)$. Then, it is not difficult to see that $z$ is well defined in the rectangle $\overline{R_{*}}$, where

$$
R_{*}:=\left(b^{\prime}, L_{0}\right) \times\left(0, S_{L}\right), \quad S_{L}:=\left(L_{0}-b^{\prime}\right)^{2} \int_{0}^{T} \frac{1}{\left(L(t)-b^{\prime}\right)^{2}} d s
$$

and, moreover,

$$
\left\{\begin{array}{l}
z_{\tau}-z_{\xi \xi}+h(\xi, \tau) z_{\xi}=0, \quad(\xi, \tau) \in R_{*}, \\
z\left(b^{\prime}, \tau\right)=y\left(b^{\prime}, t\right), \quad z\left(L_{0}, \tau\right)=0, \quad \tau \in\left(0, S_{L}\right), \\
z(\xi, 0)=y_{0}(\xi), \quad \xi \in\left(0, L_{0}\right),
\end{array}\right.
$$

where $h \in C^{0}\left(\overline{R_{*}}\right)$ and $\|h\|_{\infty}$ is bounded by a constant only depending on $N_{L}, b^{\prime}, L_{0}$ and $T$. Consequently, taking again into account Theorems 10.1 and 11.1 in 19 and the facts that $y$ satisfies 21 and $z\left(b^{\prime}, \tau\right) \equiv y\left(b^{\prime}, t\right)$, we deduce that

$$
z \in C_{\xi, \tau}^{1+\kappa, \kappa / 2}\left(\overline{R_{*}}\right),
$$


whence we get 200 .

Now, let the function $V_{L}$ be given by

$$
V_{L}(t):=y_{x}(L(t), t) \quad \forall t \in[0, T] .
$$

Obviously, $V_{L} \in C^{\kappa}([0, T])$. Furthermore, from the estimates in the proofs of Theorems 10.1 and 11.1 in $\underline{19}$, we also have

$$
\left\|V_{L}\right\|_{C^{\kappa}([0, T])} \leq C_{3}\left\|y_{0}\right\|_{\infty},
$$

where $C_{3}$ only depends on $N_{L}, L_{*}, B, \omega, T$ and $N_{0}$ and is non-decreasing with respect to $N_{L}$ and $N_{0}$.

\section{Proof of the main result}

We will first see that there exists $\varepsilon>0$ such that, if $N_{0}=\left\|y_{0}\right\|_{H_{0}^{1}\left(0, L_{0}\right)} \leq \varepsilon$, for any small $\beta>0$, there exist uniformly bounded $L_{\beta} \in C^{1}([0, T])$ and control-pairs $\left(v_{\beta}, y_{\beta}\right)$ satisfying (1)-(3) and (9). To this purpose, we will use a fixed point argument.

Thus, let $\beta>0$ and $R>0$ be given, let us set

$$
\mathcal{M}:=\left\{\ell \in C^{1}([0, T]): L_{*} \leq \ell \leq B, \quad \ell(0)=L_{0}, \quad N_{\ell}:=\left\|\ell^{\prime}\right\|_{\infty} \leq R\right\}
$$

and let us introduce the mapping $\Lambda_{\beta}: \mathcal{M} \mapsto C^{1}([0, T])$, defined as follows: for each $\ell \in \mathcal{M}$,

$$
L=\Lambda_{\beta}(\ell) \quad \text { if and only if } \quad L(t)=L_{0}-\int_{0}^{t} y_{x}(\ell(s), s) d s \quad \forall t \in[0, T],
$$

where $y$ is the solution to the linear system

$$
\left\{\begin{array}{l}
y_{t}-y_{x x}=v 1_{\omega}, \quad(x, t) \in Q_{\ell} \\
y(0, t)=0, \quad y(\ell(t), t)=0, \quad t \in(0, T), \\
y(x, 0)=y_{0}(x), \quad x \in\left(0, L_{0}\right)
\end{array}\right.
$$

and $v$ is the $\beta$-control of minimal norm in $L^{2}(\omega \times(0, T))$ associated to $\ell$, i.e. the unique solution to the following extremal problem:

$$
\left\{\begin{array}{l}
\text { Minimize } \iint_{\omega \times(0, T)}|v|^{2} d x d t \\
\text { Subject to } v \in L^{2}(\omega \times(0, T)),(25,96 .
\end{array}\right.
$$

Let us see that, if $N_{0}$ is small enough (depending on $R$ but not on $\beta$ ), we can apply Schauder's Theorem to $\Lambda_{\beta}$ in $\mathcal{M}$ and deduce the existence of a fixed point.

Obviously, $\mathcal{M}$ is a non-empty bounded, closed, convex subset of $C^{1}([0, T])$. In view of Theorem 2.1 and the strict convexity of the norm in $L^{2}(\omega \times(0, T))$, 26) possesses exactly one solution for each $\ell \in \mathcal{M}$ and the mapping $\Lambda_{\beta}$ is well-defined.

- Let us see that $\Lambda_{\beta}$ is continuous. 
Thus, let us assume that $\ell_{n} \in \mathcal{M}$ for all $n \geq 1$ and $\ell_{n} \rightarrow \ell$ in $C^{1}([0, T])$. Let us denote by $\left(v^{n}, y^{n}\right)$ the control-state pair associated to $\ell_{n}\left(v^{n}\right.$ is the solution to 26) for $\ell=\ell_{n}$ and $y^{n}$ is the corresponding state). Then, we know that

$$
v^{n}=\left.\hat{\varphi}_{n}\right|_{\omega \times(0, T)},
$$

where $\hat{\varphi}_{n}$ is the solution to (14) for $L=\ell_{n}$ and $\varphi^{T}=\hat{\varphi}_{n}^{T}$, while $\hat{\varphi}_{n}^{T}$ is the unique minimizer of $J_{\ell_{n}, \beta}$ (see 18) for the definition of this functional).

From (19), we see that $\left\|\hat{\varphi}_{n}^{T}\right\|_{L^{2}(0, \ell(T))}$ is uniformly bounded. Consequently, at least for a subsequence (again indexed by $n$ ), one has:

$$
\begin{aligned}
& \hat{\varphi}_{n}^{T} \rightarrow \hat{\varphi}^{T} \text { weakly in } L^{2}(0, \ell(T)), \\
& \hat{\varphi}_{n} \rightarrow \hat{\varphi} \text { strongly in } L^{2}(\omega \times(0, T)), \\
& \hat{\varphi}_{n}(\cdot, 0) \rightarrow \hat{\varphi}(\cdot, 0) \text { strongly in } L^{2}\left(0, L_{0}\right),
\end{aligned}
$$

where $\hat{\varphi}$ is the solution to 14 for $L=\ell$ and $\varphi^{T}=\hat{\varphi}^{T}$. In particular, this implies:

$$
\liminf J_{\ell_{n}, \beta}\left(\hat{\varphi}_{n}^{T}\right) \geq J_{\ell, \beta}\left(\hat{\varphi}^{T}\right) .
$$

Let us check that $\hat{\varphi}^{T}$ is the unique minimizer of $J_{\ell, \beta}$. This will prove that the whole sequence $\left\{\hat{\varphi}_{n}^{T}\right\}$ converges to $\hat{\varphi}^{T}$.

Thus, let $\varphi^{T} \in L^{2}(0, \ell(T))$ be given and let the functions $\varphi_{n}^{T} \in L^{2}\left(0, \ell_{n}(T)\right)$ be such that their extensions by zero converge strongly in $L^{2}(0, B)$ to the extension by zero of $\varphi^{T}$. Let us denote by $\varphi_{n}$ (resp. $\varphi$ ) the solution to (14) with $L=\ell_{n}$ and $\varphi^{T}=\varphi_{n}^{T}$ (resp. $L=\ell$ ). Then, it is clear that $\varphi_{n} \rightarrow \varphi$ strongly in $L^{2}(\omega \times(0, T))$ and $\varphi_{n}(\cdot, 0) \rightarrow \varphi(\cdot, 0)$ strongly in $L^{2}\left(0, L_{0}\right)$ and, consequently,

$$
J_{\ell_{n}, \beta}\left(\varphi_{n}^{T}\right) \rightarrow J_{\ell, \beta}\left(\varphi^{T}\right) .
$$

A direct consequence of (27) and (28) is that

$$
J_{\ell, \beta}\left(\hat{\varphi}^{T}\right) \leq \liminf J_{\ell_{n}, \beta}\left(\hat{\varphi}_{n}^{T}\right) \leq \liminf J_{\ell_{n}, \beta}\left(\varphi_{n}^{T}\right)=J_{\ell, \beta}\left(\varphi^{T}\right)
$$

and, since $\varphi^{T}$ is arbitrary, $\hat{\varphi}^{T}$ minimizes $J_{\ell, \beta}$ in $L^{2}(0, \ell(T))$.

An immediate consequence is that the controls $v^{n}$ converge strongly to the solution to 26 . Also, the states $y^{n}$ converges strongly (for instance in the $L^{2}$ sense) to the associated state $y$. Finally, from the estimates in Section 2.4, it becomes clear that $y_{x}^{n}\left(\ell_{n}(\cdot), \cdot\right) \rightarrow y_{x}(\ell(\cdot), \cdot)$ strongly in $C^{0}([0, T])$ and $\Lambda_{\beta}\left(\ell_{n}\right) \rightarrow \Lambda_{\beta}(\ell)$ strongly in $C^{1}([0, T])$.

Hence, $\Lambda_{\beta}$ is continuous.

- The mapping $\Lambda_{\beta}$ is also compact. In fact, all the $\Lambda_{\beta}(\ell)$ with $\ell \in \mathcal{M}$ belong to a fixed compact set of $C^{1}([0, T])$ (independent of $\beta$ ).

This can be seen as follows: in view of (24) and the estimates (23) (where $V_{L}$ is given by (22) and $C_{3}$ only depends on $N_{L}, L_{*}, B, \omega, T$ and $N_{0}$ ), the $\Lambda_{\beta}(\ell)$ belong to a fixed bounded set of $C^{1+\kappa}([0, T])$ for some $\kappa \in(0,1 / 2]$ that is independent of $\ell$; this, together with the compactness of the embedding $C^{1+\kappa}([0, T]) \hookrightarrow C^{1}([0, T])$, allows to conclude. 
- Finally, let us see that, if $N_{0}$ is small enough (uniformly with respect to $\beta$ ), $\Lambda_{\beta}$ maps $\mathcal{M}$ into itself. In fact, this is clear from (23): by construction, $\Lambda_{\beta}(\ell) \in C^{1}([0, T])$ and $\Lambda_{\beta}(\ell)(0)=L_{0}$; we also have

$$
\left|\Lambda_{\beta}(\ell)^{\prime}(t)\right|=\left|y_{x}(\ell(t), t)\right| \leq C_{3}\left\|y_{0}\right\|_{\infty} \leq C_{4} N_{0} \text { and }\left|\Lambda_{\beta}(\ell)(t)-L_{0}\right| \leq C_{3} T\left\|y_{0}\right\|_{\infty} \leq C_{4} T N_{0}
$$

for all $t \in[0, T]$, where $C_{3}$ and $C_{4}$ depend on $R, L_{*}, B, \omega, T$ and $N_{0}$ and are non-decreasing with respect to $N_{0}$. Therefore, if we choose $\varepsilon$ sufficiently small and $N_{0} \leq \varepsilon$, we will have

$$
\begin{aligned}
N_{0} & \leq \min \left(\frac{R}{C_{4}}, \frac{B-L_{0}}{C_{4} T}, \frac{L_{0}-L_{*}}{C_{4} T}\right), \\
\left|\Lambda_{\beta}(\ell)^{\prime}(t)\right| & \leq R \text { and } L_{*} \leq \Lambda_{\beta}(\ell)(t) \leq B \quad \forall t \in[0, T],
\end{aligned}
$$

whence the desired property $\Lambda_{\beta}(\ell) \in \mathcal{M}$ holds.

Let $L_{\beta}$ be a fixed point of $\Lambda_{\beta}$ for each $\beta>0$. Then, it is clear that $L_{\beta}$ satisfies, together with some $v_{\beta}$ and $y_{\beta},(1)-(3)$ and $(9)$. Moreover, $L_{\beta}$ and $v_{\beta}$ are uniformly bounded in $C^{1+\kappa}([0, T])$ and $L^{2}(\omega \times(0, T))$, respectively.

Consequently, our assertion is proved.

Now, at least for a subsequence, one has

$$
L_{\beta} \rightarrow L \text { strongly in } C^{1}([0, T]) \text { and } v_{\beta} \rightarrow v \text { weakly in } L^{2}(\omega \times(0, T))
$$

as $\beta \rightarrow 0$. Obviously, $L$ satisfies (1). Also, it is clear that the unique solution to (2) satisfies (3) and (4).

Hence, Theorem 1.1 is proved.

\section{Some additional comments}

In Theorem 1.1. we have established the null controllability of (1)-(3) with distributed controls, locally supported in space. It is unknown whether a similar global controllability result holds. Notice that the proof of such a result seems difficult: for general initial data in $H_{0}^{1}\left(0, L_{0}\right)$, even when $v \equiv 0$, the existence of a global in time solution to the free-boundary problem (1)-(3) fails, see $\underline{7}$.

To our knowledge, there are, at least, two other ways to prove Theorem 1.1 via a fixed point argument:

- We can work directly with $\beta=0$ and introduce other controls for 25) (not necessarily of minimal norm in $\left.L^{2}(\omega \times(0, T))\right)$. A good candidate is the control furnished by the FursikovImanuvilov method, described in 15. In this case, it can be proved that the mapping $\Lambda_{0}$ is continuous. 
- Alternatively, we can introduce a multi-valued mapping (assigning to each $\ell$ the whole family of null-controls) and, then, apply Kakutani's fixed point theorem.

On the other hand, it would be interesting to extend Theorem 1.1. at least, to the following situations:

1. Free-boundary problems for semilinear parabolic PDEs with (Lipschitz-continuous) nonlinear terms of the form

$$
\begin{gathered}
\left\{\begin{array}{l}
y_{t}-y_{x x}+f\left(y, y_{x}\right)=v 1_{\omega}, \quad(x, t) \in Q_{L}, \\
y(0, t)=0, \quad y(L(t), t)=0, \quad t \in(0, T), \\
y(x, 0)=y_{0}(x), \quad x \in\left(0, L_{0}\right),
\end{array}\right. \\
y_{x}(L(t), t)=-L^{\prime}(t), \quad t \in(0, T),
\end{gathered}
$$

2. Two-phase Stefan-like problems with the following structure:

$$
\left\{\begin{array}{l}
y_{t}-a y_{x x}=v 1_{\omega}, \quad(x, t) \in Q_{L}, \\
z_{t}-b z_{x x}=0, \quad(x, t) \in D_{L}, \\
y(0, t)=0, \quad y(L(t), t)=z(L(t), t)=0, \quad z(B, t)=0, \quad t \in(0, T), \\
y(x, 0)=y_{0}(x), \quad x \in\left(0, L_{0}\right), \\
z(x, 0)=z_{0}(x), \quad x \in\left(L_{0}, B\right), \\
\quad\left(a y_{x}-b z_{x}\right)(L(t), t)=-L^{\prime}(t), \quad t \in(0, T),
\end{array}\right.
$$

where $D_{L}$ stands for the set

$$
D_{L}=\{(x, t): x \in(L(t), B), \quad t \in(0, T)\} .
$$

3. Radially symmetric and star-shaped systems, etc.

Some of these extensions will be considered in the next future.

Finally, let us mention that the exact controllability to the free trajectories of (1)-(3) is also a challenging and very interesting question.

\section{Appendix: proof of Theorem 2.2}

Let us introduce $\psi$, with $\varphi:=e^{s \alpha} \psi$. Then

$$
\begin{gathered}
\varphi_{t}=s \alpha_{t} e^{s \alpha} \psi+e^{s \alpha} \psi_{t}, \quad \varphi_{x}=s \alpha_{x} e^{s \alpha} \psi+e^{s \alpha} \psi_{x}, \\
\varphi_{x x}=e^{s \alpha}\left(\psi_{x x}+s^{2} \alpha_{x}^{2} \psi+2 s \alpha_{x} \psi_{x}+s \alpha_{x x} \psi\right) .
\end{gathered}
$$

Taking into account that

$$
\begin{gathered}
\alpha_{x}=-\xi_{x}=-\lambda \alpha_{0, x} \xi, \quad \alpha_{x x}=-\lambda^{2} \alpha_{0, x}^{2} \xi-\lambda \alpha_{0, x x} \xi \\
\alpha_{t}=-k \frac{T-2 t}{(T-t)^{k+1} t^{k+1}}\left(e^{2 \lambda\left\|\alpha_{1}\right\|_{\infty}}-e^{\lambda \alpha_{1}(x, t)}\right)-\lambda \alpha_{0, t} \xi
\end{gathered}
$$


we see that, for $\lambda$ sufficiently large, one has:

$$
\left|\alpha_{x}\right| \leq C \lambda \xi, \quad\left|\alpha_{x x}\right| \leq C \lambda^{2} \xi, \quad\left|\alpha_{t}\right| \leq C \xi^{1+1 / k}+C \lambda \xi \leq C \lambda \xi^{3 / 2}
$$

(recall that $k \geq 2$; also, note that, here and henceforth, $\lambda$ and the constants $C$ depend on $N_{L}$ ). We also have that $\psi(x, 0) \equiv 0$ in $(0, L(0))$ and $\psi(x, T) \equiv 0$ in $(0, L(T))$.

In the new variable $\psi$, the PDE in 12 reads

$$
\left(\psi_{t}+2 s \alpha_{x} \psi_{x}\right)+\left(\psi_{x x}+s^{2} \alpha_{x}^{2} \psi\right)=-e^{-\alpha} g-s \alpha_{x x} \psi-s \alpha_{t} \psi
$$

Let us introduce the following notation:

$$
\left\{\begin{array}{l}
U \psi:=\psi_{t}+2 s \alpha_{x} \psi_{x}, \\
V \psi:=\psi_{x x}+s^{2} \alpha_{x}^{2} \psi
\end{array}\right.
$$

Then one has:

$$
U \psi+V \psi=-e^{-\alpha} g-s \alpha_{x x} \psi-s \alpha_{t} \psi
$$

whence

$$
\begin{aligned}
& \|U \psi\|_{L^{2}\left(Q_{L}\right)}^{2}+\|V \psi\|_{L^{2}\left(Q_{L}\right)}^{2}+2(U \psi, V \psi)_{L^{2}\left(Q_{L}\right)} \\
& \quad=\left\|e^{-\alpha} g+s \alpha_{x x} \psi+s \alpha_{t} \psi\right\|_{L^{2}\left(Q_{L}\right)}^{2} \\
& \quad \leq C \iint_{Q_{L}} e^{-2 \alpha}|g|^{2} d x d t+C \iint_{Q_{L}} \lambda^{4} s^{2} \xi^{3}|\psi|^{2} d x d t .
\end{aligned}
$$

After some computations, we see that

$$
\begin{aligned}
& (U \psi, V \psi)_{L^{2}\left(Q_{L}\right)}=s \iint_{Q_{L}}\left(-\alpha_{x x}\right)\left|\psi_{x}\right|^{2} d x d t \\
& \quad-\iint_{Q_{L}}\left(3 s^{3} \alpha_{x}^{2} \alpha_{x x}+2 s^{2} \alpha_{x} \alpha_{x t}\right)|\psi|^{2} d x d t \\
& \quad+\int_{0}^{T}\left(s \alpha_{x}(L(t), t)-\frac{1}{2} L^{\prime}(t)\right)\left|\psi_{x}(L(t), t)\right|^{2} d t-\int_{0}^{T} s \alpha_{x}(0, t)\left|\psi_{x}(0, t)\right|^{2} d t .
\end{aligned}
$$

Note that

$$
-\alpha_{x}^{2} \alpha_{x x}=\lambda^{4}\left|\alpha_{0, x}\right|^{4} \xi^{3}+\lambda^{3}\left|\alpha_{0, x}\right|^{2} \alpha_{0, x x} \xi^{3} \geq C \lambda^{4} \xi^{3}-C \lambda^{3} \xi^{3} \quad \text { in } \overline{Q_{L}} \backslash\left(\omega_{0} \times(0, T)\right) .
$$

On the other hand,

$$
\alpha_{x}(L(t), t) \geq C>0 \quad \text { and } \quad-\alpha_{x}(0, t) \geq C>0 \quad \text { in }(0, T) .
$$

Consequently, we get the following for all sufficiently large $s$ and $\lambda$ :

$$
\begin{aligned}
& (U \psi, V \psi)_{L^{2}\left(Q_{L}\right)} \geq C \iint_{Q_{L}}\left((s \xi) \lambda^{2}\left|\psi_{x}\right|^{2}+(s \xi)^{3} \lambda^{4}|\psi|^{2}\right) d x d t \\
& \quad+C s \lambda \int_{0}^{T}\left(\xi(L(t), t)\left|\psi_{x}(L(t), t)\right|^{2}+\xi(0, t)\left|\psi_{x}(0, t)\right|^{2}\right) d x d t \\
& \quad-C \iint_{\omega_{0} \times(0, T)}\left((s \xi) \lambda^{2}\left|\psi_{x}\right|^{2}+(s \xi)^{3} \lambda^{4}|\psi|^{2}\right) d x d t .
\end{aligned}
$$


From $(29)$ and $(30)$, we deduce that

$$
\begin{aligned}
& \|U \psi\|_{L^{2}\left(Q_{L}\right)}^{2}+\|V \psi\|_{L^{2}\left(Q_{L}\right)}^{2}+\iint_{Q_{L}}\left((s \xi) \lambda^{2}\left|\psi_{x}\right|^{2}+(s \xi)^{3} \lambda^{4}|\psi|^{2}\right) d x d t \\
& \quad+s \lambda \iint_{0}^{T}\left(\xi(L(t), t)\left|\psi_{x}(L(t), t)\right|^{2}+\xi(0, t)\left|\psi_{x}(0, t)\right|^{2}\right) d x d t \\
& \leq C\left(\iint_{Q_{L}} e^{-2 \alpha}|g|^{2} d x d t+\iint_{\omega_{0} \times(0, T)}(s \xi)^{3} \lambda^{4}|\psi|^{2} d x d t\right) \\
& \quad+C \iint_{\omega_{0} \times(0, T)}(s \xi) \lambda^{2}\left|\psi_{x}\right|^{2} d x d t .
\end{aligned}
$$

Now, we can argue as in $\underline{15}$ to get $[13)$ from (31); see also $\underline{9}$. The steps are the following:

- First, we note that

$$
\|V \psi\|_{L^{2}\left(Q_{L}\right)}^{2} \geq C \iint_{Q_{L}}(s \xi)^{-1}\left|\psi_{x x}\right|^{2} d x d t-C \iint_{Q_{L}}(s \xi)^{3} \lambda^{4}|\psi|^{2} d x d t .
$$

- Then, we see that

$$
\|U \psi\|_{L^{2}\left(Q_{L}\right)}^{2} \geq C \iint_{Q_{L}}(s \xi)^{-1}\left|\psi_{t}\right|^{2} d x d t-C \iint_{Q_{L}}(s \xi) \lambda^{2}\left|\psi_{x}\right|^{2} d x d t .
$$

- Also, we prove that, for each $\varepsilon>0$, we can find $C_{\varepsilon}$ such that

$$
\iint_{\omega_{0} \times(0, T)}(s \xi) \lambda^{2}\left|\psi_{x}\right|^{2} d x d t \leq \varepsilon \iint_{Q_{L}}(s \xi)^{-1}\left|\psi_{x x}\right|^{2} d x d t+C_{\varepsilon} \iint_{\omega \times(0, T)}(s \xi)^{3} \lambda^{4}|\psi|^{2} d x d t .
$$

Putting (31)-(34) together, we deduce that

$$
\begin{gathered}
\iint_{Q_{L}}\left((s \xi)^{-1}\left(\left|\psi_{t}\right|^{2}+\left|\psi_{x x}\right|^{2}\right)+(s \xi) \lambda^{2}\left|\psi_{x}\right|^{2}+(s \xi)^{3} \lambda^{4}|\psi|^{2}\right) d x d t \\
\quad+s \lambda \int_{0}^{T}\left(\xi(L(t), t)\left|\psi_{x}(L(t), t)\right|^{2}+\xi(0, t)\left|\psi_{x}(0, t)\right|^{2}\right) d t \\
\leq C\left(\iint_{Q_{L}} e^{-2 \alpha}|g|^{2} d x d t+\iint_{\omega \times(0, T)}(s \xi)^{3} \lambda^{4}|\psi|^{2} d x d t\right) .
\end{gathered}
$$

Finally, coming back to the original variable $\varphi$, it is very easy to obtain (13).

\section{References}

[1] Aronson, D.G., Some properties of the interface for a gas flow in porous media, in "Free Boundary Problems: Theory and Applications, Volume I", (Fasano A. and Primicerio, M. eds.), Research Notes Math., no. 78, Pitman, London, 1983.

[2] Avalos, G., Lasiecka, I., Boundary controllability of thermoelastic plates via the free boundary conditions, SIAM J. Control Optim. 38 (2000), no. 2, 337-383 (electronic).

[3] Doubova, A., Fernández-Cara, E., Some control results for sim-plified one-dimensional models of fluid-solid interaction, Math. Models Methods Appl. Sci. 15 (2005), no. 5, 783-824.

[4] Doubova, A., Fernández-Cara, E., González-Burgos, M., Zuazua, E., On the controllability of parabolic systems with a nonlinear term involving the state and the gradient, SIAM J. Control Optim. 41 (2002), no. 3, 798-819. 
[5] Fabre, C., Puel, J. P., Zuazua, E., Approximate controllability of the semilinear heat equation, Proc. Roy. Soc. Edinburgh Sect. A 125 (1) (1995) 31-61.

[6] Fasano, A., Mathematical models of some diffusive processes with free boundaries, MAT, Series A: Mathematical Conferences, Seminars and Papers, 11, Universidad Austral, Rosario, 2005.

[7] Fasano, A., Primicerio, General free-boundary problems for the heat equation, I, J. Math. Anal. Appl. 57 (1977), 694-723.

[8] Fattorini, H.O., Russell, D.L., Exact controllability theorems for linear parabolic equations in one space dimension, Arch. Rational Mech. Anal. 43 (1971), no. 4, 272-292.

[9] Fernández-Cara, E., Guerrero, S., Global Carleman Inequalities for parabolic systems and applications to controllability, SIAM Control Optm., V. 45, nº 4 (2006) pp. 1395-1446.

[10] Fernández-Cara, E., Zuazua, E., The cost of approximate controllability for heat equations: the linear case, Adv. Differential Equations, 5(2000), no. 4-6, 465-514.

[11] Friedman, A., Partial differential equations of parabolic type, Prentice-Hall, Inc., Englewood Cliffs, N.J., 1964.

[12] Friedman, A., Variational principles and free-boundary problems, John Wiley \& Sons, Inc., New York, 1982.

[13] Friedman, A. (Ed.), Tutorials in mathematical biosciences, III. Cell cycle, proliferation, and cancer, Lecture Notes in Mathematics, 1872, Mathematical Biosciences Subseries, Springer-Verlag, Berlin, 2006.

[14] Friedman, A., PDE problems arising in mathematical biology, Netw. Heterog. Media 7 (2012), no. 4, 691-703.

[15] Fursikov, A. V., Imanuvilov, O.Yu., Controllability of evolution equations, Lectures Notes Series, Vol. 34, National University, RIM, Seoul, South Korea, 1996.

[16] Gupta, S.C., The classical Stefan problem. Basic concepts, modelling and analysis, Elsevier Science B.V., Amsterdam, 2003.

[17] Hansen, S.W., Imanuvilov, O.Yu, Exact controllability of a multilayer Rao-Nakra plate with free boundary conditions, Math. Control Related Fields 1 (2011), no. 2, 189-230.

[18] Hermans, A. J., Water waves and ship hydrodynamics. An introduction, 2nd edition, Springer, Dordrecht, 2011.

[19] Ladyzhenskaya, O. A., Solonnikov, V. A., Uraltseva, N. N., Linear and quasilinear equations of parabolic type, Translations of Mathematical Monographs, Vol. 23, American Mathematical Society, Providence, R.I. 1968.

[20] Lebeau, G., Robbiano, L., Contrôle exact de l'équation de la chaleur, Comm. Partial Differential Equations 20 (1995), no. 1-2, 335-356.

[21] Liu, Y., Takahashi, T., Tucsnak, M., Sinqle input control-llability of a simplified fluid-structure interaction model, ESAIM Control Optim. Calc. Var. 19 (2013), no. 1, 20-42.

[22] Stoker, J.J., Water waves, Interscience, New York, 1957.

[23] Vázquez, J.L., The porous medium equation. Mathematical theory, Oxford Mathematical Monographs, The Clarendon Press, Oxford University Press, Oxford, 2007.

[24] Vázquez, J.L., Zuazua, E., Large time behavior for a simplified 1D model of fluid-solid interaction, Comm. Partial Differential Equations 28 (2003), no. 9-10, 1705-1738.

[25] Wrobel, L.C., Brebbia, C.A. (Eds.), Computational modelling of free and moving boundary problems, Vol. 1, Fluid flow, Proceedings of the First International Conference held 2-4 July, 1991, Southampton, U.K., Computational Mechanics Publications, Southampton, 1991. 